\title{
The control of saccade trajectories: Direction of curvature depends on prior knowledge of target location and saccade latency
}

\author{
ROBIN WALKER \\ Royal Holloway, University of London, Egham, England \\ EUGENE MCSORLEY \\ University of Reading, Reading, England \\ and \\ PATRICK HAGGARD \\ University College London, London, England
}

\begin{abstract}
Recent reports have shown that saccades can deviate either toward or away from distractors. However, the specific conditions responsible for the change in initial saccade direction are not known. One possibility, examined here, is that the direction of curvature (toward or away from distractors) reflects preparatory tuning of the oculomotor system when the location of the target and distractor are known in advance. This was investigated by examining saccade trajectories under predictable and unpredictable target conditions. In Experiment 1, the targets and the distractors appeared unpredictably, whereas in Experiment 2 an arrow cue presented at fixation indicated the location of the forthcoming target prior to stimulus onset. Saccades were made to targets on the horizontal, vertical, and principal oblique axis, and distractors appeared simultaneously at an adjacent location (a separation of $\pm 45^{\circ}$ of visual angle). On average, saccade trajectories curved toward distractors when target locations were unpredictable and curved away from distractors when target locations were known in advance. There was no overall difference in mean saccade latencies between the two experiments. The magnitude of the distractor modulation of saccade trajectory (either toward or away from) was comparable across the different saccade directions (horizontal, vertical, and oblique). These results are interpreted in terms of the time course of competitive interactions operating in the neural structures involved in the suppression of distractors and the selection of a saccade target. A relatively slow mechanism that inhibits movements to distractors produces curvature away from the distractor. This mechanism has more time to operate when target location is predictable, increasing the likelihood that the saccade trajectory will deviate away from the distractor.
\end{abstract}

The paths of saccadic eye movements are often found to be curved (Dodge, 1917; Helmholtz, 1962; Yarbus, 1967). This natural tendency for curved trajectories is greater for oblique than for horizontal or vertical saccades (Viviani \& Swensson, 1982). Furthermore, the direction and magnitude of saccade curvature can be modulated by a range of different factors. For example, in the so-called double-step task, saccades are directed initially toward the first target step but, on occasions, show strong curvature toward the second target (Findlay \& Harris, 1984; Van Gisbergen, Van Opstal, \& Roebroek, 1987). Saccades in visual search tasks

This work was funded by a grant from the Leverhulme Trust (awarded to R.W. and P.H.). The authors thank Steve Hammett for useful suggestions about the experiments described here and Jan Theeuwes and Richard Abrams for helpful comments and insights on an earlier draft. Correspondence concerning this article should be addressed to R. Walker, Department of Psychology, Royal Holloway, University of London, Egham, Surrey TW20 0EX, England (e-mail: robin.walker@, rhul.ac.uk). have been shown to deviate toward the location of competing distractors (McPeek, Han, \& Keller, 2003; McPeek $\&$ Keller, 2001), and after an erroneous saccade has been made toward a distractor, a subsequent secondary corrective saccade may be made that deviates back toward the target (McPeek \& Keller, 2001). By contrast, in other situations, saccade trajectories have been shown to deviate away from attended distractor locations. Sheliga and colleagues (Sheliga, Craighero, Riggio, \& Rizzolatti, 1997; Sheliga, Riggio, Craighero, \& Rizzolatti, 1995; Sheliga, Riggio, \& Rizzolatti, 1995) showed that saccades deviated away from a previously attended location and that the magnitude of curvature was greatest when the saccade target was in the attended hemifield. This finding was attributed to a specific process of inhibition operating within spatial attention. However, Doyle and Walker (2001) showed that prior attentional orienting is not necessary for the effect; saccades also deviated away from the location of a task-irrelevant distractor onset presented simultaneously with the saccade target (see also Tipper, Howard, \& Paul, 2001). 
The distractor modulation of saccadic, and manualreach, trajectories (Sheliga et al., 1997; Tipper et al., 2001) has been interpreted in terms of competitive processes operating within neural structures involved in the encoding of potential targets (Doyle \& Walker, 2001, 2002; McPeek \& Keller, 2001; McSorley, Haggard, \& Walker, 2004; Sheliga, Riggio, \& Rizzolatti, 1995; Tipper et al., 2001). The superior colliculus (SC) is an important neural structure involved in the control of eye movements (Sparks \& Hartwich-Young, 1989; Wurtz, 2000), and the activity of populations of neurons in its intermediate layers is thought to operate as a two-dimensional motor map encoding the metrics of potential saccade goals (Sparks \& Hartwich-Young, 1989; Wurtz, Basso, Paré, \& Sommer, $2000)$. Since the visual array is typically cluttered, there are, typically, multiple peaks of neural activity forming a salience map encoding potential saccade targets (Findlay \& Walker, 1999; McIlwain, 1986). Competitive interactions have been shown to operate between separate populations of neurons to resolve the conflict of competing target locations, thus enabling the selection of a unique saccade target (Munoz \& Istvan, 1998). This kind of competitive process is often invoked to explain saccade deviation (Doyle \& Walker, 2001, 2002; McPeek et al., 2003; McPeek \& Keller, 2001; McSorley et al., 2004; Sheliga et al., 1997; Sheliga, Riggio, et al., 1995; Sheliga, Riggio, \& Rizzolatti, 1994, 1995).

\section{Curvature Toward Distractors}

The deviation of a saccade toward a distractor (or a subsequent saccade goal) has been attributed to distractorrelated activity's being above a threshold level at the time a saccade is initiated (McPeek et al., 2003; McPeek \& Keller, 2001). In this case, the distractor-related activity is assumed to merge with the target-related activity, so the saccade is directed toward a location represented by the average of the activity in the two populations of neurons. Neurophysiological support for this account has been provided by McPeek et al., who recorded single neuron responses in the $\mathrm{SC}$ and showed that curvature toward a distractor location was associated with the increased activity of neurons encoding the distractor observed just prior to saccade onset. The magnitude of curvature was correlated with the level of distractor-related activity, with greater curvature being associated with greater neural activity at the distractor site (see also Port \& Wurtz, 2003). In addition, subthreshold microstimulation of collicular sites, prior to saccade onset, was found to produce curvature toward the stimulated location. Thus, the level of neural activity at a site representing a competing saccade target can influence the balance of activity within the collicular salience map, producing deviation toward the distractor location.

\section{Curvature Away From Distractors}

The deviation of saccades away from a distractor (or attended) location requires a different explanation. This is because, on the classical salience map model, any neu- ral activity associated with the distractor is averaged with activity associated with the target. The distractor activity can, therefore, attract the saccade trajectory but not repel it. Instead, curvature away from distractors has been attributed to a process that inhibits distractor-related activity below baseline level prior to saccade initiation (Doyle \& Walker, 2001, 2002; McSorley et al., 2004; Sheliga et al., 1994; Sheliga, Riggio, \& Rizzolatti, 1995; Tipper, Howard, \& Houghton, 2000; Tipper et al., 2001). In this case, the activity in the overall population representing the target and distractor produces a saccade vector that deviates slightly away from the inhibited (distractor) location. The link between inhibition and curvature away from distractors is supported by reversible deactivation studies in which a localized injection of a GABA agonist is placed at a location in the collicular motor map. The result of this localized increase in inhibition is the deviation of saccades in the direction away from the location represented by the injection site (Aizawa \& Wurtz, 1998; Quaia, Aizawa, Optican, \& Wurtz, 1998). However, the exact source of the inhibitory processes operating in the saccadic system, for the suppression of distractor-related activity, is not yet fully understood. Local competition within a neural representation may inhibit distractor-evoked activity but should not suppress it below the level that would be expected if no distractor were present, as curvature away would require. Instead, a different, nonlocal kind of inhibition is required, based on a top-down projection from other structures. For example, the frontal eye fields (FEFs) are known to exert an inhibitory influence on the colliculus (Schlag-Rey, Schlag, \& Dassonville, 1992) and could further inhibit distractor-related activity below baseline, resulting in the deviation of saccades away from distractors.

\section{Curvature Back to Targets}

The mechanisms described above may explain the modulation of initial saccade direction observed when a distractor is present. However, an additional mechanism has been invoked to explain how saccade direction is adjusted during the saccade to produce a trajectory that curves back toward the target. The intermediate layers of the SC are thought to encode saccade endpoint (direction and amplitude) by a center-of-activity mechanism (Sparks, 1986; Sparks \& Hartwich-Young, 1989) but are not thought to be involved in the on-line control of saccade trajectory (Quaia, Lefévre, \& Optican, 1999; Soetedjo, Kaneko, \& Fuchs, 2002). These mechanisms could explain the initial deviation of saccade direction toward or away from the distractor but not curvature back toward the saccade target. Explanations of how saccades curve back toward the target have been based on a separate extracollicular feedback loop that controls saccade trajectory online, enabling small corrections to be made, so the saccade deviates back toward the saccade goal (see McSorley et al., 2004; Quaia et al., 1999). Quaia et al. (1999) outlined a detailed model in which the feedback process is attributed to the cerebellum, which is thought to receive a separate (indirect) input from the frontal and supplementary eye 
fields, which could provide a representation of the desired saccade goal (see also McSorley et al., 2004). This provides a separate directional drive signal to the brainstem saccade generator, enabling the on-line correction of a saccade that deviates from the desired saccade target. The processes involved in the control of trajectory have been discussed elsewhere (McSorley et al., 2004; Quaia et al., 1999) and are not further considered here. The focus of the present study is the influence of distractors on the direction of initial saccade deviation and, more specifically, the factors involved in producing deviation toward or away from a competing distractor.

To summarize, it remains unclear what factors cause saccades to deviate either toward or away from a competing distractor, despite apparently similar behavioral conditions. There are a number of basic differences between the behavioral paradigms used to study curvature toward and curvature away and between the species tested (humans and monkeys). These make it difficult to see what the crucial conditions are for the distractor modulation of saccade trajectory. In general, studies reporting deviation toward a distractor have used visual search tasks, with multiple target locations, whereas deviation away from distractors has typically been observed under conditions in which target location is more predictable. The mode of saccade generation could also be an important factor. The majority of studies reporting deviation away from distractors in humans have examined the trajectories of so-called voluntary saccades (Reingold \& Stampe, 2002; Sheliga, Brown, \& Miles, 2002; Walker, Walker, Husain, \& Kennard, 2000), which require a higher level of endogenous control for their generation. An exception is a study by Doyle and Walker (2001), who observed trajectory deviations away from distractors with voluntary (endogenous) and reflexive (exogenous) saccades in humans. Saccade latency also may be an underlying factor involved in the distractor modulation of saccade curvature. Voluntary saccades, made on the basis of a symbolic cue (or in visual search), have a longer latency than do reflexive stimulus-elicited saccades (e.g., Walker et al., 2000). The increase in latency for voluntary saccades increases the likelihood that distractor-related activity will be inhibited prior to saccade initiation, so voluntary saccades are more likely to deviate away from the distractor. The importance of saccade latency in the distractor modulation of trajectory has been highlighted in a recent report that showed short-latency saccades deviating toward distractors and long-latency saccades deviating away from distractors (Theeuwes \& Godijn, 2004).

A further important factor, which forms the primary focus of the present investigation, is the use of predictable distractor and target locations in paradigms designed to investigate saccade curvature. In situations in which saccades deviate toward distractors, the distractors are presented at potential saccade target locations (McPeek et al., 2003). By contrast, in situations in which saccades deviate away from distractors, the distractors do not appear at potential saccade target locations, and target location is highly predictable in advance of stimulus onset (Doyle \& Walker, 2001, 2002; McSorley et al., 2004; Sheliga et al., 1997; Sheliga, Riggio, et al., 1995; Sheliga, Riggio, \& Rizzolatti, 1995). The difference in initial saccade direction (and curvature) may be related to the time course of distractor-related inhibition. When distractors appear at potential target locations, these locations cannot be inhibited prior to target onset. Conversely, when a distractor appears exclusively at nontarget locations, inhibition can be applied to nontarget locations prior to target onset, and activity at potential target locations may be enhanced. The top-down inhibitory mechanism postulated above can be applied in the predictable situation, but not in the unpredictable situation. Since curvature away is taken as the behavioral sign of this inhibition, saccades are more likely to deviate toward a distractor when the target and distractor locations are unpredictable. For example, in visual search paradigms (McPeek et al., 2003), inhibitory processes cannot commence until after stimulus onset, increasing the time required to suppress distractor-related activity, with the consequence that saccades deviate toward distractors. By contrast, fixed, predictable target locations (e.g., Doyle \& Walker, 2001; Sheliga, Riggio, \& Rizzolatti, 1995) would allow inhibitory processes to operate prior to stimulus onset. Saccades would then be more likely to deviate away from distractors. The prior inhibition of potential distractor locations and/or greater activity at potential target locations can result in the more intense competition between target- and distractor-related activity. The consequence of this competition is that the distractor-related activity may be inhibited below baseline levels prior to saccade initiation (McPeek et al., 2003).

The present study was performed in order to compare distractor modulation of saccade direction in human subjects when target and distractor predictability was manipulated by the presence or absence of a precue. We predicted that the precue, indicating the location of the forthcoming saccade target, should cause saccades to deviate away from distractors. By contrast, when the location of a target and a distractor is not known in advance, saccades will deviate toward distractors. A secondary consideration was the effect of saccade direction on curvature. The majority of studies showing deviation away from distractors have been restricted to saccades made to targets presented on the vertical midline, except for Sheliga, Riggio, et al. (1995; horizontal and vertical saccades) and Godijn and Theeuwes (2002; two-dimensional search array). There have been no direct comparisons of the amount of curvature for saccades made in other directions under similar conditions. This was examined, in the present study, by comparing the amount of curvature for saccades made to targets presented on the horizontal, vertical, and oblique axis. In both cases, saccade targets were peripheral stimulus onsets, and so the saccades were reflexive (or stimulus elicited), as opposed to the endogenous voluntary saccades made in the majority of studies of saccade curvature. 


\section{METHOD}

\section{Apparatus and Materials}

The presentation of stimuli and eye movement recording were controlled by two computers connected via a local Ethernet link in order to coordinate stimulus presentation and data collection. Eye movements were recorded using a head-mounted video-based eyetracker (EyeLink, Sensomotoric Instruments) with a sampling frequency of $250 \mathrm{~Hz}$. A complex diamond-shaped central fixation stimulus, with sides of $1^{\circ}$, was used and ensured that the manipulation of visual events at central fixation were comparable in both experiments (see Figure 1). In Experiment 1, this took the form of a diamond with a cross in the middle, whereas in Experiment 2, the stimulus was a more complicated overlaid diamond and square with intersecting lines (see detail in Figure 1). With the offset of two lines from the fixation stimulus, an arrow cue was produced. The saccade target took the form of a cross (x) that was $1^{\circ}$ square, with each line having a thickness of $6^{\prime}$ of arc. The distractor was an unfilled circle (o) with a diameter of $1^{\circ}$ and a line thickness of $6^{\prime}$ of arc. The stimuli were presented on a $17-$ in. $60-\mathrm{Hz}$ color monitor. A chinrest was used to minimize head movements and to maintain the viewing distance at $57 \mathrm{~cm}$ from the screen.

\section{Subjects}

Six subjects participated in both experiments: 5 of them female and 1 male in Experiment 1 (18-32 years old) and 2 of them female and 4 male in Experiment 2 (22-39 years old). All had normal or corrected-to-normal vision.

\section{Design}

The target appeared $10^{\circ}$ from fixation in one of eight positions circularly arranged to be equidistant at $45^{\circ}$ apart. The stimuli were positioned so that they lay on the cardinal (horizontal and vertical) and main diagonal (oblique) axes. The distractors, when present, appeared nonpredictably in one of the two flanking positions of the target. Thus, there were 16 distractor conditions and 8 no-distractor conditions. The distractors appeared simultaneously with the onset of the saccade target. The subjects completed four blocks of 120 trials in both experiments, producing a total of 480 trials (20 trials per condition) in each.

\section{Procedure}

Prior to each block of trials, a calibration of the subjects' eye position relative to fixed points on the monitor was performed. The calibration procedure required the subjects to saccade to nine points in succession around the screen. In order to validate the eye positions recorded, the subjects again made saccades to the same nine points in succession. If landing position deviated by more than $0.5^{\circ}$, the procedure was repeated. Once the accuracy was within $0.5^{\circ}$, a block of trials was completed.

Each trial began with the appearance of the central fixation stimulus, which was displayed initially for $300 \mathrm{msec}$, after which time lines were removed from the fixation stimulus in such a way that a nondirectional hourglass (Experiment 1) or an arrow precue (Experiment 2) remained. This ensured that the manipulation of visual events at central fixation (required for presentation of the arrow precue) was similar in both experiments. This was important, since

Experiment 1

Unpredictable targets

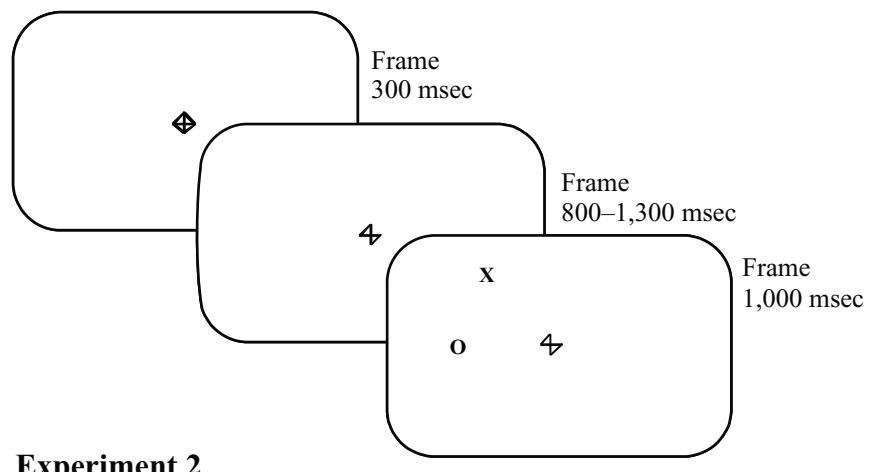

Predictable targets

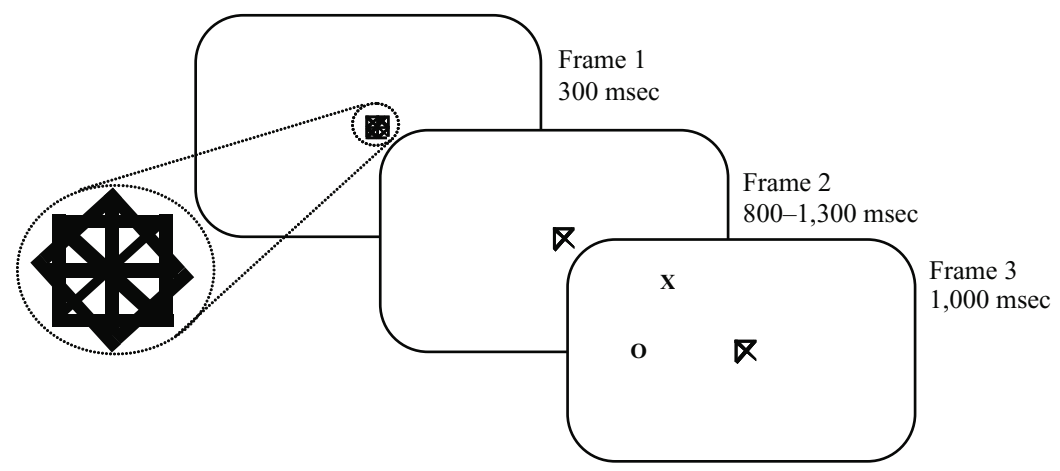

Figure 1. Example of stimulus display and time course of the trials in Experiments 1 and 2. 
saccade latency is known to be modulated by the onset or offset of a stimulus at central fixation (Ross \& Ross, 1980; Saslow, 1967). A delay of 800-1,300 msec then occurred, followed by the simultaneous onset of the target or of the target and the distractor. The onset of the target provided the subjects with the cue to move their eyes to that target location. A delay of $1,000 \mathrm{msec}$ then occurred, during which time the subjects made a saccade. The display was then blanked for an intertrial delay of $600 \mathrm{msec}$.

\section{Data Analysis}

EyeLink software identified saccade start and endpoints, using a $22^{\circ} / \mathrm{sec}$ velocity and $8,000^{\circ} / \mathrm{sec}^{2}$ acceleration criterion. The instantaneous velocity and acceleration were calculated and compared across two samples ( $8 \mathrm{msec}$ at a sample rate of $250 \mathrm{~Hz}$ ), and if either was above threshold, a saccade was detected. The endpoint of the saccade was detected when the velocity or acceleration dropped below threshold. Further analysis of saccade metrics and dynamics was carried out using software developed in MATLAB (MathWorks, Inc.).

Saccade amplitude, latency, and overall direction were derived from the eye movement records for the first saccade in each record. Amplitude was defined as the shortest distance between saccade start and endpoints (in degrees of visual angle). Latency was defined as the interval between the target onset and the saccade onset (in milliseconds). Direction was defined as the angular deviation of saccade direction taken from the initial fixation location to the final endpoint in polar coordinates.

Saccades were excluded from further analysis if (1) latencies were less than $100 \mathrm{msec}$ or greater than $500 \mathrm{msec}$, (2) the direction of the saccade was greater than $15^{\circ}$ of visual angle on either side of the target, (3) amplitudes were less than $5^{\circ}$, or (4) blinks occurred during the saccade.

Once saccades had been identified, the direction and magnitude of curvature was computed by finding the area under the curve formed by the sampled curved saccade trajectory, relative to the direct distance between fixation and landing position (see Ludwig \& Gilchrist, 2002, for a detailed description and comparison of curvature measures). In particular, at sample point $n$, the deviation perpendicular to the direction of the saccade at $n$ and $n-1$ was averaged. This average was multiplied by the distance between $n$ and $n-1$ along the direct route of the saccade. In order to normalize across the varying amplitude of saccades, the area measure was divided by the amplitude of the saccade to obtain a ratio measure of curvature per unit amplitude. Since saccade trajectories are never completely straight, the area of curvature observed in no-distractor (baseline) conditions was subtracted from that observed under distractor conditions (note that baseline curvature for each subject was calculated and subtracted for each target direction separately). Thus, the distractor modulation of trajectory reported here is in terms of the difference in curvature relative to the baseline level of curvature for saccades made to comparable target locations in no-distractor conditions. Trajectories deviating toward the distractor have been assigned positive curvature values, and those deviating away from the distractor have been assigned negative curvature values.

\section{RESULTS}

Figure 2 shows mean saccade curvature for Experiment 1 (no-cue unpredictable targets) and Experiment 2 (precue predictable targets), collapsed across target position and distractor locations. A mixed factor ANOVA was performed to examine the effect of target position (eight levels) and distractor location (two levels: counterclockwise and clockwise), with experiment as a betweensubjects factor (two levels). The ANOVA confirmed a sig-

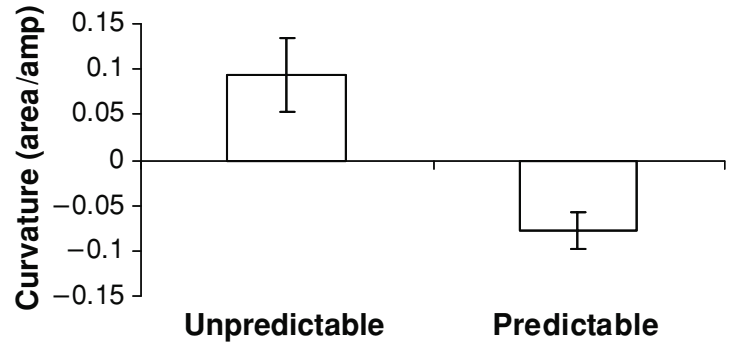

Figure 2. Mean saccade trajectory deviation for all target and distractor positions under no-cue or precue conditions. Positive values indicate curvature toward the distractor location, whereas negative values indicate curvature away from the distractor location. Error bars show 1 standard error of the mean.

nificant main effect of experiment $[F(1,5)=17.2, p<.01]$ on saccade curvature but no effect of either target position $[F(7,35)=1.2, p>.05]$ or distractor location $[F(1,5)=$ $6.6, p>.05]$ and no interaction effects $[F(7,35)<1]$. Thus, when target position was unpredictable (Experiment 1), saccades deviated toward the distractor location, and when the target was predictable (Experiment 2), saccades deviated away from the distractor location. Table 1 shows mean saccade amplitude for the two experiments, under distractor and no-distractor conditions. An ANOVA confirmed that there was no difference in saccade amplitude between experiments $[F(1,10)<1]$ or distractor conditions $[F(1,10)=4.1, p>.05]$ and no interaction effects $[F(1,10)<1]$. It is important to note that since saccade amplitude was comparable across conditions, the measure of curvature used (area under curve normalized by amplitude) cannot be influenced by the distractor modulation of saccade amplitude.

The effects of saccade direction (horizontal, vertical, and oblique) on curvature were also examined. Figures $3 \mathrm{~A}$ and $3 \mathrm{~B}$ show mean curvature grouped by horizontal, verti$\mathrm{cal}$, and oblique target locations (curvature has been collapsed across the two horizontal, two vertical, and four oblique target locations). Figure 3A shows mean curvature observed in the baseline condition, when no distractor was present. Here, the ordinate shows saccade curvature clockwise and counterclockwise of the straight trajectory. Although vertical saccades appear more curved than horizontal or oblique saccades, separate one-way ANOVAs (three levels of direction) showed no significant effect of target direction on curvature for either Experiment 1

Table 1

Mean Saccade Amplitude (in Degrees, With Standard Deviations) in the Distractor and No-Distractor Conditions in Experiments 1 and 2

\begin{tabular}{cccccc}
\hline \multirow{2}{*}{ Condition } & \multicolumn{2}{c}{ No Distractor } & & \multicolumn{2}{c}{ Distractor } \\
\cline { 2 - 3 } \cline { 5 - 6 } & $M$ & $S D$ & & $M$ & $S D$ \\
\hline Experiment 1, no cue & $9.9^{\circ}$ & $0.8^{\circ}$ & & $9.5^{\circ}$ & $0.7^{\circ}$ \\
Experiment 2, precue & $9.8^{\circ}$ & $0.9^{\circ}$ & & $9.4^{\circ}$ & $0.4^{\circ}$ \\
\hline
\end{tabular}




\section{A) No-Distractor Condition}

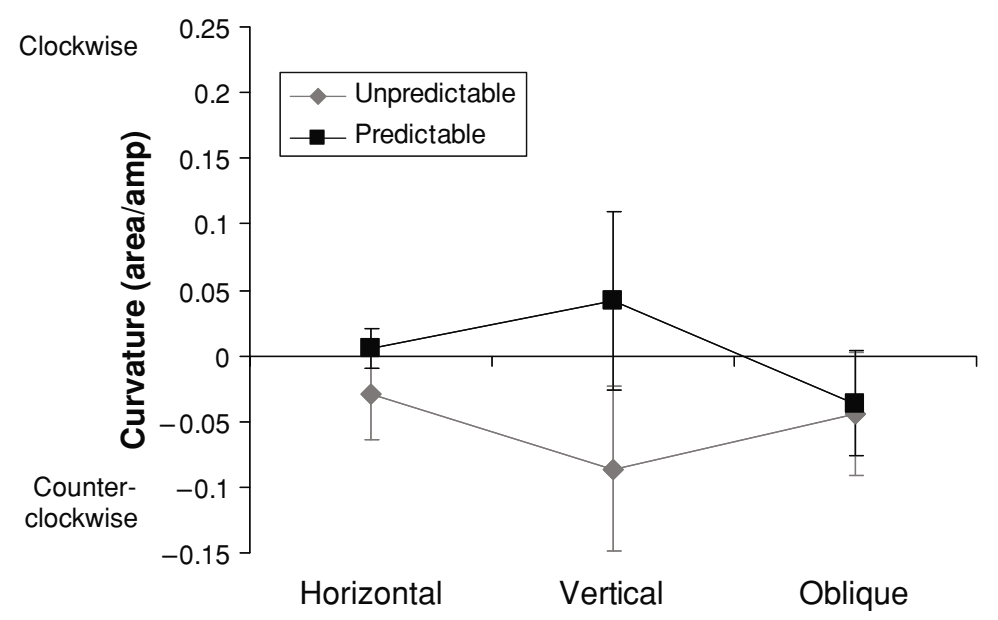

\section{B) Distractor Condition}

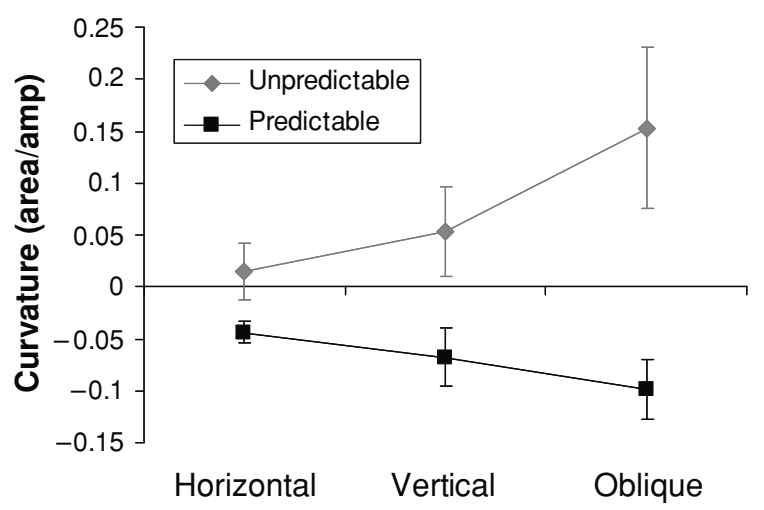

Figure 3. Mean trajectory deviation made under unpredictable (gray) or predictable (black) target conditions grouped by saccade direction: whether the saccade was made to a target shown on one of the main diagonals (obliques) or one of the cardinal axes (vertical or horizontal). The upper plot (A) shows curvature in the baseline no-distractor condition. Positive values on the ordinate show curvature clockwise of the straight target-directed trajectory, and negative values show curvature counterclockwise of this straight trajectory. The lower plot (B) shows the modulation of curvature when a distractor was presented. Positive values on the ordinate show curvature toward the distractor location, and negative values show curvature away from the distractor location.

$[F(2,10)<1]$ or Experiment $2[F(2,10)=1.4$; both $p$ s $>$ $.05]$. Figure $3 \mathrm{~B}$ shows the change in curvature when a distractor was present. The ordinate shows curvature toward $(+v e)$ and away from $(-v e)$ the distractor for each saccade direction. Oblique saccades show larger trajectory modulations in both experiments; however, separate oneway ANOVAs for each experiment showed this trend to be nonsignificant [Experiment 1, $F(2,10)=2.1$; Experiment 2, $F(2,10)=2.7$; both $p$ s $>.05]$.

Saccade latency has been shown to be an important factor in the distractor modulation of trajectory. Although the direction of curvature (toward or away from) was different in the two experiments, mean saccade latencies were almost equivalent (Experiment 1, 216 msec; Experiment 2,
$215 \mathrm{msec}$ ). Mean saccade latency was increased, however, by the presence of a distractor in both experiments (Experiment 1, no distractor $=209 \mathrm{msec}$, distractor $=223 \mathrm{msec}$; Experiment 2, no distractor $=211 \mathrm{msec}$, distractor $=$ $220 \mathrm{msec}$ ). This small remote distractor latency increase (of some 9-14 msec) was found to be significant in both cases [Experiment 1, $t(5)=3.1, p<.05$; Experiment 2, $t(5)=2.8, p<.05]$. Separate ANOVAs were performed to examine the influence of saccade direction (three levels: horizontal, vertical, and oblique) on latency (Experiment 1 , horizontal $=209 \mathrm{msec}$, vertical $=211 \mathrm{msec}$, oblique $=208 \mathrm{msec} ;$ Experiment 2, horizontal $=$ $202 \mathrm{msec}$, vertical $=210 \mathrm{msec}$, oblique $=216 \mathrm{msec}$ ). Saccade direction had no significant effect on latency in 
the no-distractor conditions for either experiment [Experiment 1, $F(2,10)<1$; Experiment 2, $F(2,10)=1.6, p>$ $.05]$. An effect of saccade direction was revealed for latency in the distractor conditions (Experiment 1, horizontal $=$ $218 \mathrm{msec}$, vertical $=223 \mathrm{msec}$, oblique $=225 \mathrm{msec}$; Experiment 2 , horizontal $=212 \mathrm{msec}$, vertical $=217 \mathrm{msec}$, oblique $=226 \mathrm{msec}$ ), but this was found to be significant only in Experiment 2 [Experiment 1, $F(2,10)=1.1, p>$ .05 ; Experiment 2, $F(2,10)=7, p<.05]$.

The relationship between saccade latency and curvature was further examined in terms of latency distributions (see Figure 4). Figure 4 shows saccade curvature as a function of latency. The latency distributions for each subject have been grouped into bins of $20 \%$ (quintiled) and then averaged across subjects. Thus, moving from left to right, the figure shows the curvature for the quickest $20 \%$ of saccades to that for the slowest $20 \%$ of saccades. The ordinate shows mean curvature toward (positive values) and away from (negative values) the distractor location. Error bars are repeated measures $95 \%$ confidence intervals. An ANOVA was performed on saccade curvature, with latency as a within-subjects factor (five levels: quintiles) and experiment as a between-subjects factor. Significant main effects of latency $[F(4,40)=5.1, p<.05]$ and experiment $[F(1,10)=16.1, p<.01]$ were found, and there was no interaction effect $[F(4,40)=1.2, p>.05]$. With unpredictable targets, saccades curved toward distractor locations when latency was short and became less curved as latency increased, with curvature away from distractors being observed for the slowest $20 \%$ of saccades. With pre- dictable targets, saccades deviated away from the distractor across all latency intervals.

\section{DISCUSSION}

The present study was performed to examine the direction of saccade trajectory deviation observed when a competing distractor was presented along with the saccade target. It was predicted that saccade trajectories would deviate toward distractors with unpredictable targets (where distractors appear at potential target locations) and away from distractors when target location was known in advance. The distractor modulation of saccade trajectory was examined under unpredictable (no-cue) and predictable (precue) target conditions. In both cases, distractors appeared at the location adjacent to the target on the majority of trials, and the amount of curvature was compared with that observed under no-distractor (baseline) conditions. On average, saccade trajectories curved toward distractors with unpredictable targets and curved away from distractors when target location was known in advance. There was no overall difference in mean latency between the two experimental conditions, although there was a trend for curvature away from distractors to be associated with a longer latency (Theeuwes \& Godijn, 2004). Finally, there was no difference in the overall pattern of results for the different saccade directions, although curvature under distractor conditions was greater for oblique saccades. Our study has shown that human subjects show deviation toward distractors under unpredictable target conditions and

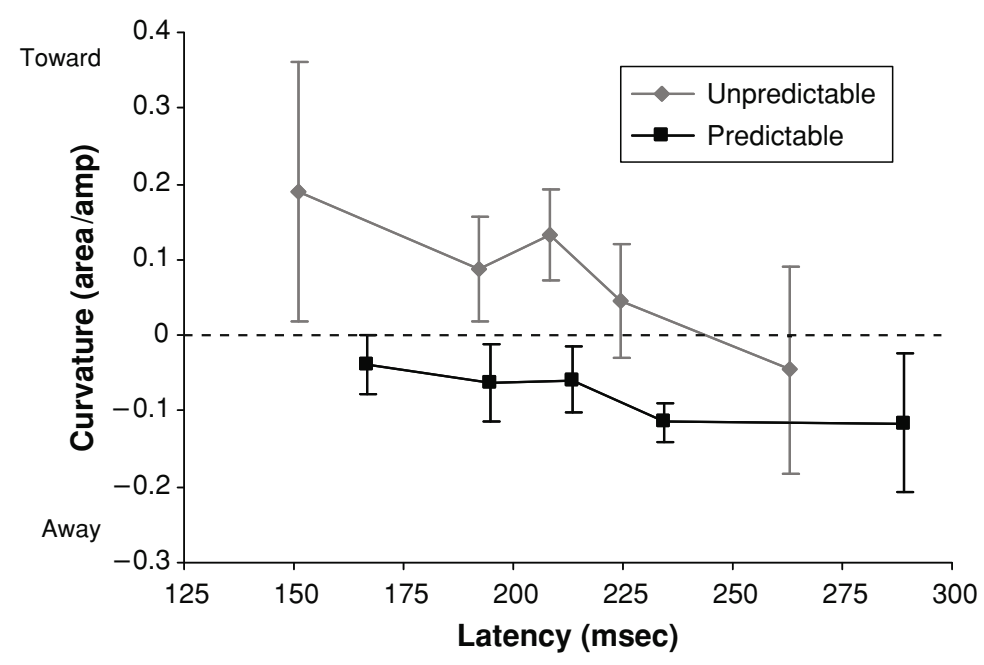

Figure 4. Saccade curvature plotted as a function of quintiled saccade latency. The curvature elicited from the quickest $20 \%$ of saccades to the slowest $\mathbf{2 0 \%}$ of saccades is shown progressively from left to right. The abscissa shows latency in milliseconds, and the ordinate shows curvature in terms of deviation toward (positive values) or away from (negative values) the distractor. Saccade curvature when target location was unpredictable is shown in gray, and that found when target location was predictable is shown in black. Error bars are repeated measures $95 \%$ confidence intervals, which allow for comparison within each distribution. 
away from distractors with predictable target locations, across all target directions. Differences in the direction of saccade deviation (toward or away from distractors) does not, therefore, depend on species (humans/monkeys), the mode of saccade generation (voluntary/reflexive), or saccade direction. These findings will be discussed in terms of competitive interactions operating within the saccadic system for the selection of a saccade goal.

Models of saccade curvature have been based on the idea of competitive interactions operating in a salience map encoding potential saccade targets (Godijn \& Theeuwes, 2002; McPeek et al., 2003; McPeek \& Keller, 2001; McSorley et al., 2004; Tipper et al., 2000). The intermediate layers of the SC are thought to form such a salience map, with populations of neurons with large and broadly overlapping receptive fields forming a topographic representation of saccade direction (Mcllwain, 1991). A saccade is directed to the location represented by the area of maximum activity within this map (see Findlay \& Walker, 1999). When separate populations of neurons associated with a target and a distractor are active, the overall activity profile can result in a saccade's being directed to an average of the two locations, as in the global effect (Findlay, 1982). Similar explanations have been advanced to explain the curvature toward a distractor location observed with visual search (McPeek et al., 2003), although one important difference here is that the target and the distractor are not located on the same axis and it is the initial saccade direction that is modulated by the competition between the target and the distractor. When distractor-related activity remains above surrounding baseline activity when a saccade is initiated, there is a small shift in the activity of the overall population of neurons associated with the distractor, and the saccade will deviate toward the distractor location. By contrast, if the distractor-related activity is inhibited and is below baseline level prior to saccade initiation, there is a small shift in the overall activity profile away from the distractor location, producing a deviation of initial saccade direction away from the distractor (Aizawa \& Wurtz, 1998; McPeek et al., 2003). In both cases, curvature back toward the saccade target may be attributed to a separate process involved in directing the eye back toward the target in midflight with a separate representation of the desired saccade goal (McSorley et al., 2004; Quaia et al., 1999).

We propose that the level of activity at potential distractor sites varies between predictable and unpredictable target conditions. The relationship observed between saccade latency and direction of curvature (cf. Theeuwes \& Godijn, 2004) is consistent with this interpretation and with explanations that emphasize the time course of inhibitory processes involved in saccade target selection (Godijn \& Theeuwes, 2004; Theeuwes \& Godijn, 2004). When the target location is known in advance (precued condition), inhibitory processes may be applied prior to stimulus onset: Activity at the target location is heightened, and that at potential distractor locations is reduced. In particular, the activity at potential distractor locations may be below baseline in the predictable target condition, due to broad inhibition for nontarget locations prior to the onset of the saccade goal. Activity at saccade target locations may be enhanced, by presumed top-down processes, when target location is predictable. We have previously shown that the distractor-related inhibition has very coarse spatial tuning and is relatively insensitive to the spatial separation between target and distractor (McSorley et al., 2004). Such broad, inhibitory processes can be applied prior to distractor onset under conditions in which target location is known in advance. The FEFs provide a plausible substrate for activation of the desired target location and spatially broad inhibition of nontarget locations. The FEFs send projections to the intermediate layers of the SC that activate neurons encoding the desired saccade vectors while inhibiting activity of neurons at other locations (Schlag-Rey et al., 1992). In addition, complex lateral interactions are known to exist in the SC, with separate populations exciting one another when close and inhibiting each other when remote (Munoz \& Istvan, 1998). We propose that the local interactions within the SC sum additively with the top-down modulation. The timing is offset, however, since the top-down component has a head start in the predictable target condition only. The saccade deviates away from distractors, under predictable target conditions, because the top-down inhibitory processes may allow distractor locations to be inhibited prior to stimulus onset. The consequence is that the intensity of the competition between target and distractor is greater when target location is predictable and, so, distractor-related activity is more likely to be suppressed below baseline prior to saccade initiation. Prior to target and distractor onset, inhibition is assumed to be applied equally to all potential distractor sites. Once the distractor location is made apparent (following stimulus onset), inhibition can then be lifted from other potential distractor locations and applied more firmly to the distractor itself. The result is an overall shift in the neural activity of the population of neurons away from the distractor location and deviation of initial saccade direction away from that location.

Under unpredictable target conditions, the same competitive processes operate, but the target and the distractor cannot be discriminated in advance of stimulus onset, and so this preparatory inhibition cannot occur. In this situation, the inhibitory processes take longer to suppress distractor-related activity below the surrounding baseline activity. As a result, the difference between the activity at the distractor location (presumably quite high due to transient onset) and that at other, surrounding nontarget locations will remain high for a longer period of time than under predictable target conditions. Thus, there is a greater likelihood of saccades deviating toward the distractor location when target location is unpredictable.

A broadly similar model has recently been advanced by Godijn and Theeuwes (2004; Theeuwes \& Godijn, 2004), who examined the relationship between saccade curvature and inhibition of return (IOR). IOR refers to the delay observed when a saccade is made to a peripheral location, 
where attention was directed covertly (Posner, 1980), before being disengaged (see Klein, 2000). The time course of IOR is relatively slow and depends critically on covert attention's having visited a location before moving on. It is thought to serve as a device that facilitates optimal visual search behavior. Godijn and Theeuwes (2004) provide evidence against IOR's being the factor involved in producing trajectory deviation by demonstrating a dissociation in the time course of task-irrelevant distractors for IOR and saccade curvature. Trajectory deviations occurred at short delays, and IOR at long delays. Thus, IOR and the prior orienting of covert attention to a location are not thought to underlie saccade curvature. They propose a model similar to ours to account for saccade curvature, with inhibition that is applied to distractor locations in a saccade map producing a deviation away from a distractor. IOR is attributed to inhibition in a separate, but strongly related, system termed the preoculomotor attention map that provides input to the saccade map, where the final stages of oculomotor planning take place.

A related issue is the involvement of exogenous (reflexive) and endogenous (voluntary) covert attention (Müller \& Rabbitt, 1989) in producing distractor-related curved saccade trajectories. In Experiment 1 of the present study, distractors and targets were peripheral onsets, and it might be argued that they summoned attention exogenously, whereas in Experiment 2, the use of the arrow precue may have enabled an endogenous shift of attention to the target location prior to target onset. It might, therefore, be suggested that the exogenous orienting of attention is involved in producing curvature toward distractors, whereas endogenous orienting of attention may be required to produce curvature away from distractors. There are a number of reasons why a degree of caution is required with such attentional interpretations of saccade curvature. First, in both experiments here, the target was a peripheral stimulus onset that appeared simultaneously with the distractor, both of which would be expected to exogenously capture attention, and in both situations the saccades can be regarded as being stimulus elicited. Second, Doyle and Walker (2001) showed that both stimulus-elicited (reflexive) and arrow-cued (voluntary) saccades curved away from task-irrelevant distractors. In both cases, distractors appeared simultaneously with the onset of the peripheral saccade target (reflexive saccades) or the central arrow cue (voluntary saccades). Endogenous attention could not, therefore, be oriented to the target location in advance of stimulus onset, but saccades were found to deviate away from distractor locations. Although it is possible that endogenous attention was oriented to the target location in our Experiment 2, this cannot be the sole factor involved in producing curvature away from distractors (Doyle \& Walker, 2001). We have argued that saccades curved away from distractors in Doyle and Walker's (2001) study and in our Experiment 2 because of the use of predictable target conditions. Prior knowledge of target location enables preparatory inhibition to be applied to distractor locations, which results in saccades' deviating away from distractor locations.
A further issue to be considered is the relationship between distractor modulation of saccade landing position (endpoint) and curvature. Intuitively, it might be expected that if saccade amplitude is influenced by a distractor, this could influence the measure of saccade curvature used. In our studies of saccade curvature, we have endeavored to reduce the effects of amplitude modulation in biasing the measure of curvature (the area under the curve) by normalizing in terms of saccade amplitude and also by applying an amplitude and direction exclusion criterion (Doyle \& Walker, 2001, 2002; McSorley et al., 2004). In the present experiment, there was no difference in saccade amplitude observed under distractor and no-distractor conditions. Furthermore, McSorley et al. examined the relationship between distractor-to-target spatial separation on saccade landing position and curvature. It was found that there was no relationship between saccade landing position and curvature (see also Port \& Wurtz, 2003). The independence of landing position and trajectory supports the view that separate processes are involved in selecting the goal for the saccade and in determining the trajectory (Port \& Wurtz, 2003; Quaia et al., 1999). The initial direction of a saccade (either toward or away from a distractor) reflects the combined activity of the target and distractor representations, and the curvature back toward the target involves a separate feedback system that provides an on-line error correction mechanism that may involve a separate input from the FEFs or cerebellum to the brainstem saccade generator (Port \& Wurtz, 2003; Quaia et al., 1999).

One final observation from the present study concerns the equivalence of mean saccade latency observed in the two experiments reported here. Intuitively, cuing the target location might be expected to reduce saccade latency. It is perhaps surprising that mean saccade latency was not reduced in Experiment 2 (precue condition), since it was possible for a saccade to be prepared in advance of target onset. In practice, the precue improved saccade accuracy (fewer direction errors) but had no influence on mean latency. This is consistent with previous studies that have shown that advance knowledge of spatial selection does not influence saccade latency (Findlay \& Walker, 1999, and subsequent commentaries). So the precue condition made the subjects more accurate (reduced erroneous saccades made to distractor onsets) and resulted in shorter latencies (anticipations) but did not influence mean saccade latency, as might be predicted. We conclude that it is not the latency of saccades that is the key factor in the distractor modulation of saccade curvature, but the time course of the competitive processes involved in selecting a saccade target, which can be modulated by subtle differences in experimental conditions, such as the use of predictable target conditions.

\section{REFERENCES}

AizaWA, H., \& Wurtz, R. H. (1998). Reversible inactivation of monkey superior colliculus: I. Curvature of saccadic trajectory. Journal of Neurophysiology, 79, 2082-2096.

Dodge, R. (1917). The laws of relative fatigue. Psychological Review, 24, 89-113. 
Doyle, M., \& Walker, R. (2001). Curved saccade trajectories: Voluntary and reflexive saccades curve away from irrelevant distractors. Experimental Brain Research, 139, 333-344.

DoYle, M., \& WALKER, R. (2002). Multisensory interactions in saccade target selection: Curved saccade trajectories. Experimental Brain Research, 142, 116-130.

FINDLAY, J. M. (1982). Global processing for saccadic eye movements. Vision Research, 22, 1033-1045.

Findlay, J. M., \& Harris, L. R. (1984). Small saccades to doublestepped targets moving in two dimensions. In A. G. Gale \& F. Johnson (Eds.), Theoretical and applied aspects of eye movement research (pp. 71-78). Amsterdam: Elsevier, North-Holland.

FINDLAY, J. M., \& WALKER, R. (1999). A model of saccade generation based on parallel processing and competitive inhibition. Behavioral \& Brain Sciences, 22, 661-721.

Godisn, R., \& Theeuwes, J. (2002). Parallel programming of saccades: Evidence for a competitive inhibition model. Journal of Experimental Psychology: Human Perception \& Performance, 28, 1039-1054.

Godisn, R., \& Theeuwes, J. (2004). The relationship between inhibition of return and saccade trajectory deviations. Journal of Experimental Psychology: Human Perception \& Performance, 30, 538-554.

Helmholtz, H. von (1962). Helmholtz's treatise on physiological optics. New York: Dover.

Klein, R. M. (2000). Inhibition of return. Trends in Cognitive Sciences, 4, 138-147.

LudwiG, C. J. H., \& Gilchrist, I. D. (2002). Measuring saccade curvature: A curve-fitting approach. Behavior Research Methods, Instruments, \& Computers, 34, 618-624.

McIlwaIN, J. T. (1986). Point images in the visual system: New interest in an old idea. Trends in Neurosciences, 9, 354-358.

McIlWAIN, J. T. (1991). Distributed spatial coding in the superior colliculus: A review. Visual Neuroscience, 6, 3-13.

McPeek, R. M., Han, J. H., \& Keller, E. L. (2003). Competition between saccade goals in the superior colliculus produces saccade curvature. Journal of Neurophysiology, 89, 2577-2590.

McPeeK, R. M., \& Keller, E. L. (2001). Short-term priming, concurrent processing, and saccade curvature during a target selection task in the monkey. Vision Research, 41, 785-800.

McSorley, E., Haggard, P., \& WAlker, R. (2004). Distractor modulation of saccade trajectories: Spatial separation and symmetry effects. Experimental Brain Research, 155, 320-333.

Müller, H. J., \& RabBitT, P. M. A. (1989). Reflexive and voluntary orienting of visual attention: Time course of activation and resistance to interruption. Journal of Experimental Psychology: Human Perception \& Performance, 15, 315-330.

Munoz, D. P., \& IstVan, P. J. (1998). Lateral inhibitory interactions in the intermediate layers of the monkey superior colliculus. Journal of Neurophysiology, 79, 1193-1209.

Port, N. L., \& Wurtz, R. H. (2003). Sequential activity of simultaneously recorded neurons in the superior colliculus during curved saccades. Journal of Neurophysiology, 90, 1887-1903.

Posner, M. I. (1980). Orienting of attention. Quarterly Journal of Experimental Psychology, 32, 3-25.

Quaia, C., Aizawa, H., Optican, L. M., \& Wurtz, R. H. (1998). Reversible inactivation of monkey superior colliculus: II. Maps of saccadic deficits. Journal of Neurophysiology, 79, 2097-2110.

Quaia, C., Lefévre, P., \& Optican, L. M. (1999). Model of the control of saccades by superior colliculus and cerebellum. Journal of Neurophysiology, 82, 999-1018.

Reingold, E. M., \& Stampe, D. M. (2002). Saccadic inhibition in voluntary and reflexive saccades. Journal of Cognitive Neuroscience, 14, 371-388.

Ross, L. E., \& Ross, S. M. (1980). Saccade latency and warning signals:
Stimulus onset, offset, and change as warning events. Perception \& Psychophysics, 27, 251-257.

SASLOW, M. G. (1967). Effects of components of displacement-step stimuli upon latency for saccadic eye movement. Journal of the Optical Society of America, 57, 1024-1029.

Schlag-Rey, M., Schlag, J., \& Dassonville, P. (1992). How the frontal eye field can impose a saccade goal on superior colliculus neurons. Journal of Neurophysiology, 67, 1003-1005.

Sheliga, B. M., Brown, V. J., \& Miles, F. A. (2002). Voluntary saccadic eye movements in humans studies with a double-cue paradigm. Vision Research, 42, 1897-1915.

Sheliga, B. M., Craighero, L., Riggio, L., \& Rizzolatti, G. (1997). Effects of spatial attention on directional manual and ocular responses. Experimental Brain Research, 114, 339-351.

Sheliga, B. M., Riggio, L., Craighero, L., \& Rizzolatti, G. (1995) Spatial attention-determined modifications in saccade trajectories. NeuroReport, 6, 585-588.

Sheliga, B. M., Riggio, L., \& Rizzolatti, G. (1994). Orienting of attention and eye movements. Experimental Brain Research, 98, 507 522

Sheliga, B. M., Riggio, L., \& Rizzolatti, G. (1995). Spatial attention and eye movements. Experimental Brain Research, 105, 261-275.

Soetedjo, R., Kaneko, C. R. S., \& Fuchs, A. F. (2002). Evidence against a moving hill in the superior colliculus during saccadic eye movements in the monkey. Journal of Neurophysiology, 87, 27782789.

SPARKS, D. L. (1986). Translation of sensory signals into commands for control of saccadic eye movements: Role of primate superior colliculus. Physiological Reviews, 66, 118-171.

Sparks, D. L., \& HARTwiCh-Young, R. (1989). The deep layers of the superior colliculus. In R. H. Wurtz \& M. E. Goldberg (Eds.), The neurobiology of saccadic eye movements (pp. 213-255). Amsterdam: Elsevier.

Theeuwes, J., \& GodiJn, R. (2004). Inhibition-of-return and oculomotor interference. Vision Research, 44, 1485-1492.

Tipper, S. P., Howard, L. A., \& Houghton, G. (2000). Behavioral consequences of selection from neural population codes. In S. Monsell \& J. Driver (Eds.), Control of cognitive processes: Attention and performance XVIII (pp. 223-245). Cambridge, MA: MIT Press.

Tipper, S. P., Howard, L. A., \& PAul, M. A. (2001). Reaching affects saccade trajectories. Experimental Brain Research, 136, 241-249.

Van Gisbergen, J., Van Opstal, A. J., \& Roebroek, J. G. H. (1987). Stimulus-induced midflight modification of saccade trajectories. In J. K. O’Regan \& A. Lévy-Schoen (Eds.), Eye movements: From physiology to cognition (pp. 27-36). Amsterdam: Elsevier.

Viviani, P., \& Swensson, R. G. (1982). Saccadic eye movements to peripherally discriminated visual targets. Journal of Experimental Psychology: Human Perception \& Performance, 8, 113-126.

Walker, R., Walker, D., Husain, M., \& Kennard, C. (2000). Control of voluntary and reflexive saccades. Experimental Brain Research, 130, $540-544$.

Wurtz, R. H. (2000). Vision for the control of movement. In M. S. Gazzaniga (Ed.), Cognitive neuroscience: A reader (pp. 341-365). Malden, MA: Blackwell.

Wurtz, R. H., Basso, M. A., Paré, M., \& Sommer, M. A. (2000). The superior colliculus and the cognitive control of movement. In M. S. Gazzaniga (Ed.), The new cognitive neurosciences (2nd ed., pp. 573587). Cambridge, MA: MIT Press.

Yarbus, A. L. (1967). Eye movements and vision (B. Haigh, Trans.). New York: Plenum.

(Manuscript received November 17, 2003; revision accepted for publication March 7, 2005.) 